\title{
Numerical Simulation of Slurry flow in horizontal pipe based on CFD-DEM
}

\author{
Jinchao Fan ${ }^{1}$, Benchun $\mathrm{Yao}^{1}, \mathrm{Yi} \mathrm{Hao}^{2}$, Shimin $\mathrm{Zhang}^{1}{ }^{*}$, and Xiaoxiao Zhu ${ }^{1}$ \\ ${ }^{1}$ China University of Petroleum-Beijing, No.18 Fuxue Road, Changping District Beijing, China \\ ${ }^{2}$ China COMAC Shanghai Aircraft Design and Research Institute, 5188 jinke road, zhangjiang, pudong new area, Shanghai, China
}

\begin{abstract}
In this paper, we propose a novel pipeline cleaning method utilizing slurry. The reason why slurry can be used for pipeline cleaning is that the collisions between the particles and the contaminant in the pipe wall can enhance the cleaning effect. A slurry with polypropylene particles embedded in water is used to cleaning a horizontal pipe is conducted to realize this method. Because the flow characteristics of the slurry is crucial for the cleaning process, it is valuable to conduct a simulation and investigate the influence of several different factors including the convey velocity and the particle size. A 3D CFD-DEM model has been established. The indicators including pressure loss, particle accumulation level at the top of the pipe are choses to characterize the slurry flow and the influence of convey velocity and particle size has been investigated accordingly. In addition, an effective method is proposed to determine the critical convey velocity for each size of the particle.
\end{abstract}

\section{Introduction}

Pipeline is an important tool for industrial production. It plays a very important role in the transportation of goods including food, water, and oil\&gas etc ${ }^{[1]}$. Contaminant will generate inside the pipe wall, especially in the longdistance pipeline, which will not only cause pollution to the goods but also increase the flow resistance. In severe cases, it will even leads to suspension accident ${ }^{[2]}$. Therefore, the pipeline needs to be cleaned regularly. The traditional pipeline cleaning method can be divided into chemical cleaning and physical cleaning. Chemical cleaning needs the use of the pharmacy which may lead the pollution of the environment. Physical cleaning mainly includes waterjet cleaning, cleaning PIG, and mechanical cleaning etc. The waterjet cleaning needs complex equipment leading the cleaning cost to be very high. The cleaning PIG cannot get through the pipe which has a complex geometry. The mechanical cleaning may damage the pipe wall. In short, each physical cleaning method has its own obvious disadvantage. The slurry which carries particles has a good cleaning effect on the contaminant due to the continuous collision of the particles with the dirt inside the pipe during the flow.

In the process of pipeline cleaning using slurry, the flow characteristics is an important factor which affects the cleaning result a lot. The flow characteristics of the slurry could be roughly divided into three stages, namely stationary bed, sliding bed and suspension ${ }^{[3]}$. In order to enhance the cleaning, the flow is expected to reach the state of suspension as far as possible to ensure effective collision between particles and contaminant inside the pipe. However, it results in a very high flow velocity and great pressure loss as well as an increasing cost of the cleaning process. Therefore, a research on the flow characteristics of the slurry is a necessity to make the slurry-based pipeline cleaning possible. Specifically, the role played by the parameters such as particle density and diameter, flow velocity should be investigated.

In order to conduct an elementary research, water is used as the carrier because it is cheap, easy available and environment friendly. Because suspension slurry is preferred, the polypropylene particle is chosen as its density is closs to the carrier.

In the research of slurry flow characteristics, most slurries are sedimentary. The particles are heavier than the water ${ }^{[4-5]}$. In recent years, the flow characteristics of a slurry composed of light particles has gradually attracted attention. It mainly includes research on the ice slurry transport ${ }^{[6]}$, pipeline transport of agricultural slurry such as wheat straw ${ }^{[7]}$. In the research of the slurry composed of polypropylene particles, Denis Edelin ${ }^{[8]}$ used experimental methods to study the flow characteristics of the slurry. In his experiment the particle diameters are less than $1 \mathrm{~mm}$, however, the particles which have a diameter more than $1 \mathrm{~mm}$ are not studied.

In this paper, the computational fluid dynamics and discrete element method coupling calculation method (CFD-DEM) is used to simulate the flow characteristics of slurry formed by polypropylene particles with diameters of $1 \mathrm{~mm}$ and above. The influence of flow velocity and particle diameter on the flow characteristics of slurry has been study, and according to the simulation results, the critical flow velocity of the slurry which is suitable for pipeline cleaning has been obtained.

\footnotetext{
*Corresponding author: zsm7481976@163.com
} 


\section{CFD-DEM model}

\subsection{Governing equations for carrier phase}

In the CFD-DEM model, the carrier is considered as the continuous phase, and the governing equations of the carrier are sloved by the CFD. As mentioned above, water is used as the carrier, and it could be properly treated as Newtonian fluid and incompressible in our case. So, the governing equations for the carrier should be continuity equation and momentum equation, namely,

$$
\frac{\partial\left(\rho_{f}\right)}{\partial t}+\nabla\left(\rho_{f} V_{f}\right)=0
$$

and

$$
\frac{\partial\left(\rho_{f} V_{f}\right)}{\partial t}+\nabla\left(\rho_{f} V_{f} V_{f}\right)=-\nabla p+\nabla\left(\tau_{f}+\tau_{t}\right)+\rho_{f} g+F_{f p},
$$

where $\rho_{f}$ is the density of the fluid, $V_{f}$ is the average velocity of the fluid at the particle location, $p$ is the pressure of the fluid at the particle location, $F_{f p}$ is the fluid-particle interaction source term, $\tau_{f}$ is the viscous stress tensor, $\tau_{t}$ is the turbulent stress tensor. The turbulence is modeled by the standards $k-\mathcal{E}$ $\operatorname{model}^{[9]}$.

\subsection{Governing equations for particle phase}

In this paper, the particle is considered as the discrete element and the translational motion of the particles is described by the second law of Newton ${ }^{[10]}$

$$
m_{p} \frac{d V_{p}}{d t}=F_{f}+F_{f p}+F_{C}
$$

where $m_{p}$ is the mass of the particle, $V_{p}$ is the velocity of the particle, $F_{f}$ is the floating weight of the particle, $F_{f p}$ is the liquid-particle interaction source term, $F_{C}$ is the contact force of the particle.

\subsubsection{The fluid-solid interaction}

$$
F_{f p}=F_{S}+F_{M}+F_{D}+F_{A}
$$

where $F_{S}$ ie the Saffman lift force, $F_{M}$ is the Magnus lift force, $F_{D}$ is the drag force, and $F_{A}$ is the add mass force.

The Saffman lift force $F_{S}$ is modeled as ${ }^{[9,11]}$

$$
\begin{aligned}
& F_{S}=1.615 d^{2} \sqrt{\frac{\rho_{f} \mu_{f}}{\left\|\omega_{f}\right\|}}\left(V_{f}-V_{p}\right) \times \omega_{f} f\left(\operatorname{Re}_{p}, \operatorname{Re}_{s}\right) \\
& f\left(\operatorname{Re}_{p}, \operatorname{Re}_{s}\right)=\left\{\begin{array}{l}
0.0524 \sqrt{\alpha \operatorname{Re}_{p}} \operatorname{Re}_{p} \geq 40 \\
(1-0.3314 \sqrt{\alpha}) e^{-0.1 \operatorname{Re}_{p}}+0.3314 \sqrt{\alpha} \operatorname{Re}_{p}<40
\end{array}\right.
\end{aligned}
$$

where $d$ is the particle diameters, $\mu_{f}$ is the viscosity of the fluid, $\omega_{f}$ is the angular velocity of the fluid, the particle Reynolds number is defined as $\operatorname{Re}_{p}=d \rho_{f} V_{p} / \mu_{f}$, and the rotational Reynolds number is $\operatorname{Re}_{s}=d \rho_{f} \omega_{f} / \mu_{f}$, and $\alpha$ is the ratio of rotational Reynolds number to particle Reynolds number $\alpha=0.5 \operatorname{Re}_{s} / \operatorname{Re}_{p}=d \omega_{f} / V_{p}$.

The Magnus lift force $F_{M}$ is modeled as ${ }^{[9,12]}$

$$
F_{M}=\left(\frac{\pi}{8}\right) \rho_{f} d^{3} \omega_{r} \times V_{r}
$$

where $\omega_{r}$ is the relative angular velocity between the particle and the fluid, $V_{r}$ is the relative velocity between the particle and the fluid.

The drag force $F_{D}$ is modeled as ${ }^{[9,13]}$

$$
F_{D}=\frac{1}{8} \pi d^{2} C_{d} \rho_{f}\left\|V_{f}+V_{f}^{\prime}-V_{p}\right\|\left(V_{f}+V_{f}^{\prime}-V_{p}\right) C_{f}^{-\left(3.7-0.65 e^{\left(1.5-\log _{10}^{\left(\mathrm{Re}_{p}\right)}\right)_{2}}\right)}
$$

where $V_{f}$ is the average velocity of the fluid at the particle location, $V_{f}^{\prime}$ is the speed fluctuation of the fluid at the particle location, $V_{p}$ is the velocity of the particle, $C_{d}$ is the drag coefficient, $C_{f}$ is the volume fraction of the fluid, $\operatorname{Re}_{p}$ is particle Reynolds number.

The add mass force $F_{A}$ is modeled as ${ }^{[9,14]}$

$$
F_{A}=-0.5 \rho_{f} V_{p} \frac{d\left(V_{p}-V_{f}\right)}{d t} .
$$

\subsubsection{Particle-particle and particle-wall interaction}

The interaction between two particles can be modeled by the tangential force and normal force ${ }^{[15]}$,as illustrated in Fig.1 

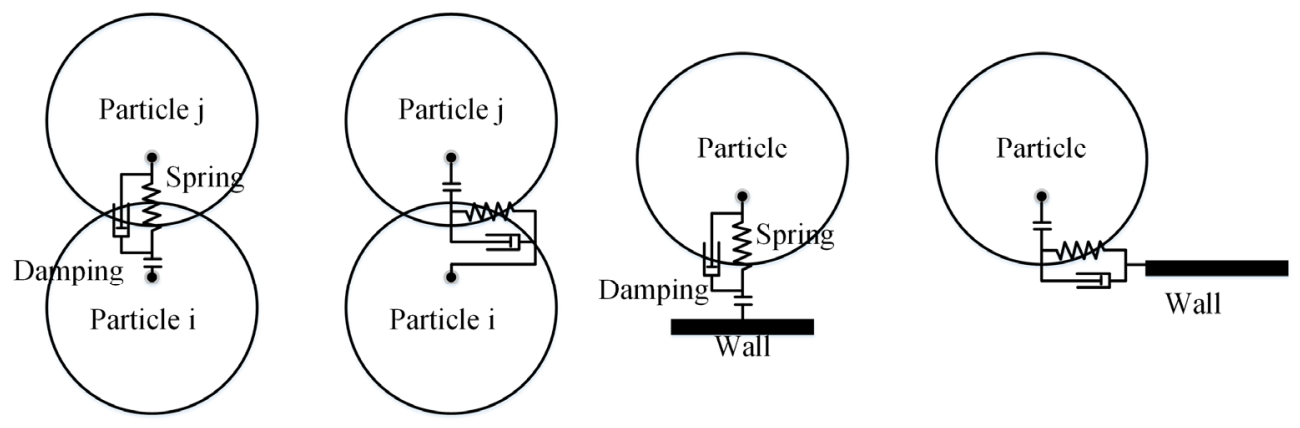

Fig. 1 The contact force of the particle ${ }^{[15]}$

The normal force $\vec{F}_{n j}$ is modeled as

$$
\vec{F}_{n i j}=\left(-k_{n} \beta^{\frac{3}{2}}-\eta_{n} \vec{G} \vec{n}\right) \vec{n}
$$

The tangential force $\vec{F}_{t i j}$ is modeled as

$$
\vec{F}_{t i j}= \begin{cases}-k_{t} \delta-\eta_{t} \vec{G}_{c t} & \left|\vec{F}_{t i j}\right| \leq \mu\left|\vec{F}_{n i j}\right| \\ \mu\left|\vec{F}_{n i j}\right| \vec{t} & \left|\vec{F}_{t i j}\right|>\mu\left|\vec{F}_{n i j}\right|\end{cases}
$$

where the $k, \eta$ is the spring stiffness and the damping coefficient, $\vec{G}$ is the relative velocity, $\beta$ is the normal displace of the contact point, $\delta$ is the tangential displace of the contact point, $\mu$ is the friction coefficient, the tangential unit vector is modeled as

$$
\vec{t}=\frac{\vec{G}_{c t}}{\left|\vec{G}_{c t}\right|}
$$

In this paper, all of the particles are of the same material and diameter, so according to the Hertz contact theory the normal spring stiffness is modeled as

$$
k_{n}=\frac{\sqrt{2 r} E}{3\left(1-v^{2}\right)},
$$

and the tangential spring stiffness is modeled according to the Mindlin-Dersiewicz contact theory

$$
k_{t}=\frac{2 \sqrt{2} G}{(2-v)} \alpha^{1 / 2},
$$

where $E$ is the elastic modulus of the particle, $G$ is the shear modulus of the particle, and $v$ is the poisson's ratio of the particle.

The damping coefficient of the normal and tangential are calculated by:

$$
\eta_{n}=2 \sqrt{m_{p} k_{n}}, \text { and } \eta_{t}=2 \sqrt{m_{p} k_{t}} .
$$

\section{Equations and mathematics} DEM software is listed in Table 1.

Table 1 The set up in the DEM software

\section{Result and discussion} concentration distribution in the outlet. particle size on the flow characteristic. $3 \mathrm{~m} / \mathrm{s}, 3.5 \mathrm{~m} / \mathrm{s}, 4 \mathrm{~m} / \mathrm{s}$ respectively.

\subsection{The influence of convey velocity} research the flow characteristics of the slurry which is composed of the Polypropylene particle. A 3D computation model is constructed with diameter of 50 $\mathrm{mm}$ and length of $1000 \mathrm{~mm}$. The model is discreted by hexahedron mesh, and the number of the whole grid is 21543. The fluid phase equation was calculated using the ANSYS FLUENT ${ }^{\odot} 15.0$ and the particle motion equation was calculated using DEM software. In the process of the simulation, the particles are randomly generated in the inlet. The particle information in the

\begin{tabular}{|c|c|c|}
\hline Material & particle & pipeline \\
\hline density & $910 \mathrm{~kg} / \mathrm{m}^{3}$ & $7850 \mathrm{~kg} / \mathrm{m}^{3}$ \\
\hline $\begin{array}{c}\text { Shear } \\
\text { modulus }\end{array}$ & $3.158 \times 10^{8} \mathrm{pa}$ & $8.077 \times 10^{11} \mathrm{pa}$ \\
\hline $\begin{array}{c}\text { Poisson's } \\
\text { ratio }\end{array}$ & 0.4103 & 0.3 \\
\hline
\end{tabular}

In this section, the flow characteristics of the slurry is analyzed according to the simulation. The influence of the convey velocity and particle size on the flow characteristics have been analyzed, and the flow characteristics of the slurry is represented by the

Firstly, the slurry with particles of $1 \mathrm{~mm}$ in diameter is used to investigate the influence of the convey velocity on the flow characteristics. Then particles of five diameters of $1 \mathrm{~mm}, 1.5 \mathrm{~mm}, 2 \mathrm{~mm}, 2.5 \mathrm{~mm}$, and 3 $\mathrm{mm}$ were selected for the research of the influence of

In the groups of simulation, the particle generation rate at the inlet keeps the same, and the convey velocity has been chosen as $1 \mathrm{~m} / \mathrm{s}, 1.5 \mathrm{~m} / \mathrm{s}, 2 \mathrm{~m} / \mathrm{s}, 2.5 \mathrm{~m} / \mathrm{s}$,

The convey velocity is an important factor affecting the slurry flow. An increase of convey velocity will lead to increase of the output pressure of the pumping equipment which will result in more energy consumption. In this paper, we choose particle of $1 \mathrm{~mm}$ 
in diameter to research the influence of convey velocity on flow characteristics as well as the pressure loss.

Fig. 2 shows the relationship between pressure loss and convey velocity. It can be observed that the pressure loss increase with convey velocity. In addition, pressure loss seems to increase faster with the convey velocity increases. Therefore, one can conclude that while ensuring the cleaning effect it is necessary to reduce the convey velocity as soon as possible in order to reduce the capacity of the pumping equipment.

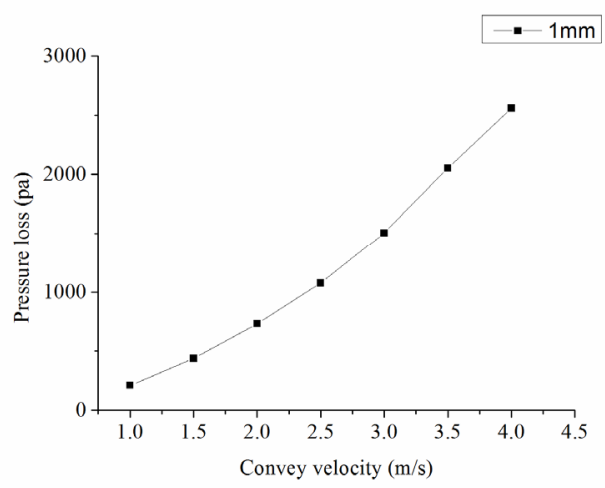

The particle concentration in the outlet is an apparent feature which can be used to characterize the flow status. Fig. 3 shows the particle concentration contour in the outlet. It can be observed from Fig. 3 that slurry flow could be grouped to two categories. When the convey velocity is less than $2 \mathrm{~m} / \mathrm{s}$, most of the particles accumulate at the top, and only few of the particles are suspended in the lower part of the pipeline. It implies that the particle concentration is denser at the top than anywhere else. In other cases, the dense zone shifts to pipeline center as convey velocity increases, and it means that the particle and the fluid gradually well mixed.

In order to intuitively display the level of the particle accumulation at the top for different convey velocity, a scale factor ' $C$ ' defined as the ratio of particle concentration above the pipe center at the $0.8 \mathrm{R}$ position to the average concentration of the pipe outlet, namely to characterize the particle concentration.

$$
C=\frac{i_{0.8 R}}{i_{\text {out }}},
$$

Fig. 2 The pressure loss of the slurry

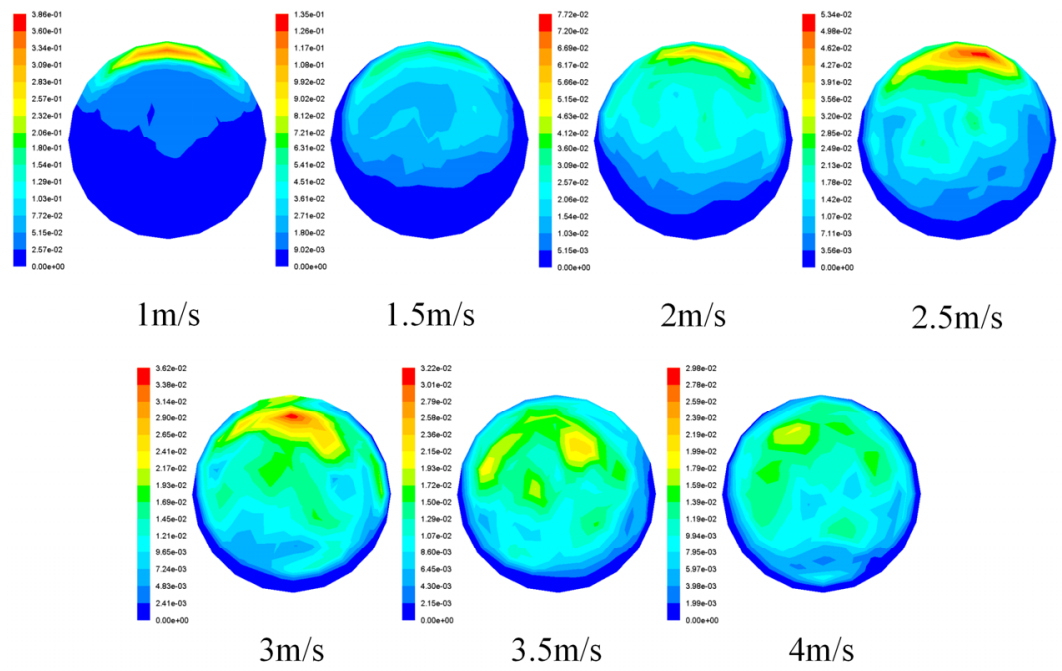

Fig. 3 Pipeline outlet particle concentration distribution contour

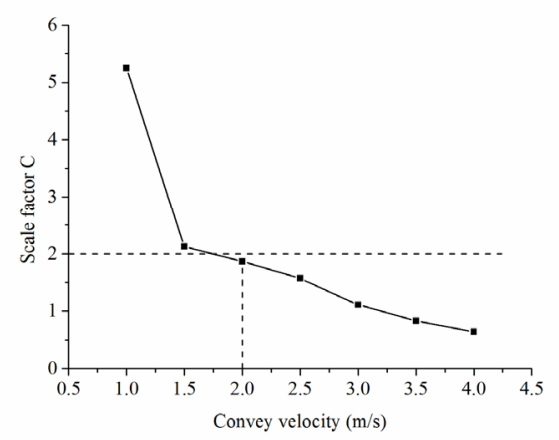

Fig. 4 The relationship of characterized concentration with convey velocity
Fig. 4 shows the curve of the scale factor $\mathrm{C}$ as a function of the slurry convey velocity. The scale factor shows a significant decrease with increasing convey velocity, and this indicates that the level of particle accumulation at the top is gradually weakened. This is consistent with the phenomenon in Fig 3. However, it can be observed from Fig. 2 that as the slurry convey velocity increases, the pressure loss is also significantly increased, which will lead to higher requirements for pumping equipment. A benchmark that $\mathrm{C} \sim 2$ was determined so that the convey velocity is desirable for pipeline cleaning. The curve in Fig. 4 shows that as the velocity increases from the $1 \mathrm{~m} / \mathrm{s}$ to the $1.5 \mathrm{~m} / \mathrm{s}, \mathrm{C}$ decreases significantly, and this suggests that the mixing state of the slurry has been significantly improved. When the velocity is increased above the $2 \mathrm{~m} / \mathrm{s}, \mathrm{C}$ is less than 2 . 
Therefore, for the slurry with a diameter of $1 \mathrm{~mm}$ the velocity of $2 \mathrm{~m} / \mathrm{s}$ could be as the critical velocity for pipeline cleaning.

\subsection{The influence of particle size}

The particle size is another factor which might affect the pipeline cleaning. At the same velocity, the increasment of the particle size can make the particle achieve higher kinetic energy, but the flow characteristic will also be changed. In this paper, particles of five different particles including diameters of $1 \mathrm{~mm}, 1.5 \mathrm{~mm}, 2 \mathrm{~mm}, 2.5 \mathrm{~mm}, 3$ $\mathrm{mm}$ are chosen to determine how the particle size affects the slurry flow. In the simulations, the mass generation rate of different size particles are kept the same, and the flow characteristic of the five different diameter particles

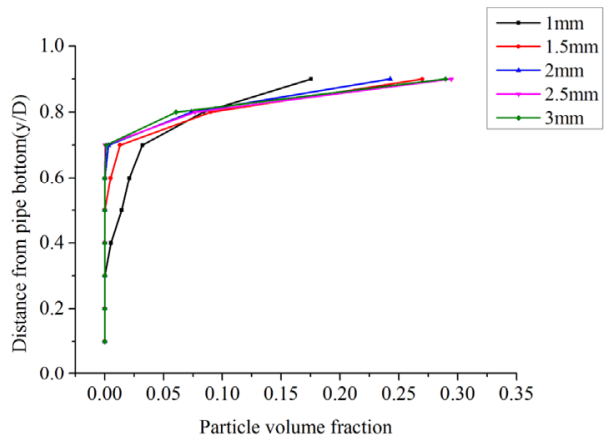

$1 \mathrm{~m} / \mathrm{s}$ at different velocity are obtained. The particle generation rate at the inlet is listed in Tab. 2 .

Table. 2 The number generated rate at the inlet

\begin{tabular}{|c|c|c|c|c|c|}
\hline $\begin{array}{c}\text { diameter } \\
(\mathrm{mm})\end{array}$ & 1 & 1.5 & 2 & 2.5 & 3 \\
\hline $\begin{array}{c}\text { Generation } \\
\text { rate }(/ \mathrm{s})\end{array}$ & $\begin{array}{c}13500 \\
0\end{array}$ & $\begin{array}{c}4000 \\
0\end{array}$ & 16875 & 8640 & 5000 \\
\hline
\end{tabular}

It can be seen from Sec. 4.1 that the particles tend to accumulate at the top of the pipe when convey velocity is low. In order to research how the particle size affects the accumulation level at the low convey velocity, the flow characteristic of the slurry which composed of different particle diameter was firstly researched at the velocity of $1 \mathrm{~m} / \mathrm{s}$ and $1.5 \mathrm{~m} / \mathrm{s}$. The particle concentration distribution curves at the outlet for the different particles were shown in the Fig. 5.

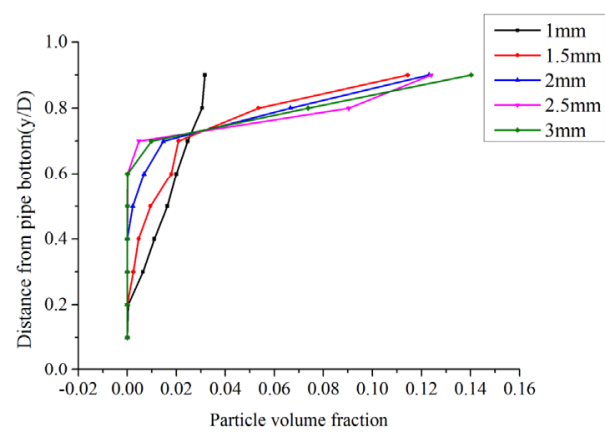

$1.5 \mathrm{~m} / \mathrm{s}$

Fig. 5 Concentration profile of outlet at velocity of $1 \mathrm{~m} / \mathrm{s}$ and $1.5 \mathrm{~m} / \mathrm{s}$

It can be observed from the Fig. 5 that in the case of the velocity is $1 \mathrm{~m} / \mathrm{s}$, all particles show the phenomenon of accumulation at the top, but the level of the accumulation has a significantly enhance with the particle size increased. When the velocity increases to $1.5 \mathrm{~m} / \mathrm{s}$ the accumulation level of the particle which has the diameter of $1 \mathrm{~mm}$ has a significantly reduce compared to the other size of the particles. This proves that the $1 \mathrm{~mm}$ diameter particles can be easier mixed than the larger particles.

In order to directly show how the particle size affects the flow characteristic of the slurry, in this section the scale factor ' $\mathrm{C}$ ' of all particles at different velocity are obtained from the simulation results, and the curves are shown in the Fig. 6.

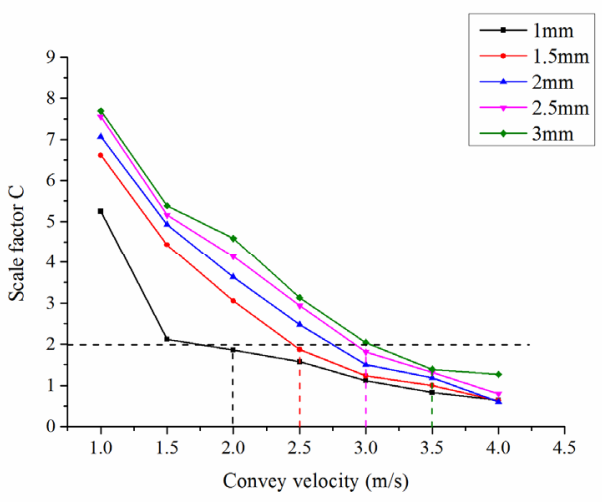

Fig. 6 Curve of the scale factor of slurry with different diameter particles as a function of convey velocity

It can be observed from the Fig. 6 that as the velocity increases the scale factor of all different particles has a significant reduce, at the same velocity the particles which have a low diameter have a lower scale factor, this proves that it can be easier mixed. According to the judging criteria proposed in the section 4.1, the critical velocity for pipeline cleaning of all different particles were obtained, and the results were shown in the Tab. 3 . 
The results in the Tab. 3 show that increasing the particle size can make the critical velocity have a significant increase, and this leads a higher requirement of the equipment. Therefore, when using the slurry for pipeline cleaning, it is necessary to reduce the diameter of the particles as much as possible while ensuring the cleaning effect. In this paper, the cleaning effect was not mentioned, the research on this issue will be completed in the subsequent work and refer to the results of this paper.

Table. 3 Critical velocity of slurry of different diameters

\begin{tabular}{|c|c|c|c|c|c|}
\hline diameter $(\mathrm{mm})$ & 1 & 1.5 & 2 & 2.5 & 3 \\
\hline velocity $(\mathrm{m} / \mathrm{s})$ & 2 & 2.5 & 3 & 3 & 3.5 \\
\hline
\end{tabular}

\section{Conclusion}

1 The convey velocity of the slurry is an important factor of the flow characteristic. As the velocity increases, in the one hand the level of the particle accumulation at the top gradually decreases, in the other hand the pressure loss gradually increases. According to the standard proposed in this paper, for the particles with diameter of $1 \mathrm{~mm}$ the critical velocity for pipeline cleaning is $2 \mathrm{~m} / \mathrm{s}$. 2 The size of the particle in the slurry is another important factor of the slurry flow characteristic. In the case of the same velocity, the larger-diameter particle exhibits a more serious tendency to accumulate at the top of the pipe and the critical convey velocity which can be used for the pipeline cleaning is higher. Therefore when using the slurry for pipeline cleaning the particle size should be reduced as much as possible while ensuring the cleaning effect.

\section{References}

1. Zhu X, Zhang S, Li X, et al. Numerical simulation of contact force on bi-directional pig in gas pipeline: At the early stage of pigging[J]. Journal of Natural Gas Science and Engineering, 2015, 23:127-138.

2. Zhu X, Wang W, Zhang S, et al. Experimental Research on the Frictional Resistance of FluidDriven Pipeline Robot with Small Size in Gas Pipeline[J]. Tribology Letters, 2017, 65(2):49.

3. Doron P, Barnea D . Flow pattern maps for solidliquid flow in pipes[J]. International Journal of Multiphase Flow, 1996, 22(2):273-283..
4. Wilson K C, Addie G R, Sellgren A, et al. Slurry Transport Using Centrifugal Pumps[M]// Slurry transport using centrifugal pumps. 2006.

5. Newitt D M . Hydraulic conveying of solids in horizontal pipes $[\mathrm{J}]$. Trans. Instn chem. Engrs, 1955..

6. Mellari S. Experimental investigations of ice slurry flows in horizontal pipe based on monopropylene glycol[J]. International Journal of Refrigeration, 2016, 65: 27-41..

7. Vaezi M , Katta A K , Kumar A . Investigation into the mechanisms of pipeline transport of slurries of wheat straw and corn stover to supply a biorefinery $[\mathrm{J}]$. Biosystems Engineering, 2014, 118(1):52-67..

8. Edelin D, Czujko P-C, Castelain C, et al. Experimental determination of the energy optimum for the transport of floating particles in pipes[J]. Experimental Thermal and Fluid Science, 2015, 68: 634-643.

9. Uzi A, Levy A. Flow characteristics of coarse particles in horizontal hydraulic conveying[J]. Powder Technology, 2018, 326: 302-321.

10. Brosh T, Kalman H, Levy A, et al. DEM-CFD simulation of particle comminution in jet-mill[J]. Powder Technology, 2014, 257: 104-112.

11. Mei R. An approximate expression for the shear lift force on a spherical particle at finite reynolds number[J]. International Journal of Multiphase Flow, 1992, 18(1):145-147.

12. Rubinow S I, Keller J B. The transverse force on a spinning sphere moving in a viscous fluid[J]. Journal of Fluid Mechanics, 1961, 11(3):447-459.

13. Felice R D . The voidage function for fluid-particle interaction systems $[\mathrm{J}]$. International Journal of Multiphase Flow, 1994, 20(1):153-159.

14. Maxey M R. The motion of small spherical particles in a cellular flow field[J]. Physics of Fluids (00319171), 1987, 30(7):1915-1928.

15. Tsuji Y, Tanaka T, Ishida T. Lagrangian Numerical Simulation of Plug Flow of Cohesionless Particles in a Horizonal Pipe[J]. Powder Technology, 1992, 71(3):239-250. 